\title{
ON A CONVERGENCE PROBLEM
}

\section{G. SHAPIRO}

Research Problem 25 [1] reads as follows:

If $a_{1}<a_{2}<\cdots$ are positive integers, if $C$ is compact, and if $\sin a_{n} x \rightarrow 0$ for all $x$ in $C$, prove that the convergence must actually be uniform.

The following example shows that the "theorem" is false.

Let

$$
a_{n}=2^{n} \quad(n=1,2, \cdots) ;
$$

let $C$ be the compact set of points

$$
(0, \pi, \pi / 2, \pi / 4, \pi / 8, \cdots) \text {. }
$$

Obviously $\sin a_{n} x \rightarrow 0$ for each $x$ in $C$, but for any $\epsilon<1$ and any positive integer, $N$, there is an $x$ in $C$ (namely, $x=\pi / 2^{N+2}$ ) such that

$$
\left|\sin a_{N+1} x\right|=|\sin \pi / 2|=1>\epsilon,
$$

so that the convergence is not uniform.

\section{REFERENCE}

1. A. Shields, Research Problem 25. A convergence problem, Bull. Amer. Math. Soc. vol. 60 (1954) p. 589.

Aircraft Armaments, Inc.

Received by the editors January 21, 1955. 